\title{
EXTENDING INFINITELY MANY TIMES ARITHMETICALLY COHEN-MACAULAY AND GORENSTEIN SUBVARIETIES OF PROJECTIVE SPACES
}

\author{
E. BALLICO
}

\begin{abstract}
We give examples of infinitely extendable (not as cones) arithmetically Cohen-Macaulay and arithmetically Gorenstein subvarieties of projective spaces and which are not complete intersections. The proof uses the computation of the dimension of the Hilbert scheme of codimension 2 subschemes of projective spaces due to G. Ellingsrud and of arithmetically Gorenstein codimension 3 subschemes due to J. O. Kleppe and R.-M. Miró-Roig.
\end{abstract}

\section{INTRODUCTION}

We recall the following classical definition (classical at least since [1, 18, 20, 21, 22, 23 ).

Definition 1.1. Let $X \subset \mathbb{P}^{n}$ be an integral and non-degenerate variety. See $\mathbb{P}^{n}$ as a hyperplane of $\mathbb{P}^{n+1}$. We say that $X$ is extendable if there is an integral and non-degenerate variety $X_{1} \subset \mathbb{P}^{n+1}$ such that $X_{1} \cap H=X$ (scheme-theoretic intersection) and $X_{1}$ is not a cone with base $X$ and vertex $p \in \mathbb{P}^{n+1} \backslash H$. In this case we say that $X_{1}$ is an extension or an 1-extension of $X$. Fix an integer $r \geq 2$. We say that $X$ is $r$-extendable if there are integral and non-degenerate varieties $X_{i} \subset \mathbb{P}^{n+i}, 1 \leq i \leq r$, with $X_{1}$ an extension of $X$ and $X_{i}, 2 \leq i \leq r$, an extension of $X_{i-1}$. We say that $X$ is infinitely extendable if it is $r$-extendable for all positive integers $r$. If $X$ is smooth (resp. locally a complete intersection) we say that $X$ is smoothly $r$-extendable (resp. $r$-extendable as locally complete intersection) if it we may take smooth (resp. locally a complete intersection) the varieties $X_{1}, \ldots, X_{r}$ appearing in the definition of $r$-extendability.

Complete intersections are infinitely extendable (just extends their minimal equations) and smooth complete intersections are infinitely smoothly extendable.

Let $r$ be any positive integer. A vector bundle $E$ on $\mathbb{P}^{n}$ is said to be $r$-extendable if there are a degree one embedding $j: \mathbb{P}^{n} \hookrightarrow \mathbb{P}^{n+r}$ and a vector bundle $F$ on $\mathbb{P}^{n+r}$ such that $E \cong j^{*}(F) . \quad E$ is said to be infinitely extendable if it is $r$-extendable for all $r$. A. N. Tyurin proved that a finite rank vector bundle on $\mathbb{P}^{n}$ is infinitely extendable if and only if it is a direct sum of line bundles (4, 5, 6, 15, 16, 20, and his result was extended to other varieties instead of a projective space (14]). Results of this type are called Babylonian towers. Zero-loci with codimension $a$ of rank $a$ vector bundles on $\mathbb{P}^{n}$ are locally a complete intersections. H. Flenner

2010 Mathematics Subject Classification. 14N05.

Key words and phrases. extensions of embedded varieties; cones; arithmetically CohenMacaulay varieties; arithmetically Gorenstein.

The author was partially supported by MIUR and GNSAGA of INdAM (Italy). 
proved that locally complete intersections which are infinitely extendable as locally complete intersections are complete intersections ([5, Theorem 2], 9]). The nonexistence (except complete intersections) of infinitely extendable locally complete intersection (except global complete intersection) easily implies the corresponding result for vector bundles.

Theorem 1.2. Fix integers $n \geq x+2 \geq 4$. There are infinitely extendable arithmetically Cohen-Macaulay integral schemes $X \subset \mathbb{P}^{n}$ with $X$ of codimension $x$ and not a complete intersection. For $x \geq 3$ there is $X$ which is arithmetically Gorenstein.

A codimension 2 arithmetically Cohen-Macaulay scheme $X \subset \mathbb{P}^{n}$ is Gorenstein if and only if it is a complete intersection ([13. Example 4.1.11(c)]). Thus we cannot require that $X$ is Gorenstein for $x=2$ and exclude the complete intersections.

There are classical non-extendability results for varieties of degree $3([21])$ and degree 4 ([18]), which come from a complete classification of all such low degree varieties. With some assumptions on the singularities there are classifications for degree 5 ([19]) and up to degree 10 ([8] ). No such results should be true in general, without also restricting the singularities of the extensions, not just of the variety. Very interesting non-extension results use the normal bundle $N_{X}$ and $h^{0}\left(N_{X}(-1)\right)-n-1$ is conjecturally an upper bound for the number of extensions, as parenthetically asked in [3, middle of the first page], which would extend a non-extendability theorem of S. Lvovsky (12), but we think that it is essential to assume something also on the singularities of the extensions. By [9] or [5, Theorem 2] it is not sufficient to assume that the singularities are locally complete intersections.

\section{The PROOF}

Proof of Theorem 1.2: Any extension of an arithmetically Cohen-Macaulay scheme of positive dimension is arithmetically Cohen-Macaulay and hence all its extensions are arithmetically Cohen-Macaulay. We start with an integral arithmetically Cohen-Macaulay $X$ which is not a cone. We will extend it to $W \subset \mathbb{P}^{n+1}$ with $W$ integral and not a cone. $W$ would be arithmetically Cohen-Macaulay and hence the construction may be iterated starting with $W$. After $r$ steps we get that $X$ is $r$-extendable. Since this is true for all $r>0, X$ is infinitely extendable in the sense of our Definition 1.1. For arithmetically Cohen-Macaulay schemes to be a complete intersection is a property of their minimal free resolution. Thus an extension $Y_{1}$ of an arithmetically Cohen-Macaulay scheme $Y$ of positive dimension is a complete intersection if and only if $Y$ is a complete intersection. Thus in each step we may omit any check that the extension is not a complete intersection.

(a) Assume $x=2$ with $n \geq 3$. Fix positive integers $s \geq 2, n_{1 i}, 1 \leq i \leq s$, and $n_{2 i}, 1 \leq i \leq s-1$, such that $\sum_{i=1}^{s-1} n_{2 i}=\sum_{i=1}^{s} n_{1 i}$. We call $\left\{n_{i j}\right\}$ these data $s, n_{1 i}$ and $n_{i j}$. Let $\mathcal{U}_{n}\left(n_{i j}\right)$ denote the set of all codimension 2 arithmetically Cohen-Macaulay schemes with free resolution

$$
0 \rightarrow \oplus_{i=1}^{s-1} \mathcal{O}_{\mathbb{P}^{n}}\left(-n_{2 i}\right) \rightarrow \oplus_{i=1}^{s} \mathcal{O}_{\mathbb{P}^{n}}\left(-n_{1 i}\right) \rightarrow \mathcal{I}_{X} \rightarrow 0
$$


By [7. Th. 2] the set $\mathcal{U}_{n}\left(n_{i j}\right)$ is a non-empty open and irreducible subset of the Hilbert scheme $\operatorname{Hilb}\left(\mathbb{P}^{n}\right)$ of $\mathbb{P}^{n}$ of dimension

$$
\begin{aligned}
& 1+\sum_{n_{2 i} \geq n_{1 j}}\left(\begin{array}{c}
n_{2 i}-n_{1 j}+n \\
n
\end{array}\right)+\sum_{n_{1 j} \geq n_{2 i}}\left(\begin{array}{c}
n_{1 j}-n_{2 i}+n \\
n
\end{array}\right) \\
& -\sum_{n_{2 i} \geq n_{2 j}}\left(\begin{array}{c}
n_{2 i}-n_{2 j}+n \\
n
\end{array}\right)-\sum_{n_{1 j} \geq n_{1 i}}\left(\begin{array}{c}
n_{1 j}-n_{1 i}+n \\
n
\end{array}\right)
\end{aligned}
$$

For instance the case $s=2$ corresponds to complete intersection codimension 2 schemes. Call $\psi_{n}\left(n_{i j}\right)$ the integer appearing in (2). For instance $\psi_{n}\left(n_{i j}\right)=$ $1+6(n+1)-4-9=6 n-6$ for $s=3, n_{11}=n_{12}=n_{13}=2$ and $n_{21}=n_{22}=3$, the case corresponding to codimension 2 degree 3 varieties (fully classified by [21] and which are always cones for $n \geq 5$ ).

See $\mathbb{P}^{n}$ as a hyperplane $H$ of $\mathbb{P}^{n+1}$. Let $\mathcal{U}_{n+1, H}\left(n_{i j}\right)$ the set of all $W \in \mathcal{U}_{n+1}\left(n_{i j}\right)$ such that no irreducible component of $W_{\text {red }}$ is contained in $H$. Note that $W \cap H \in$ $\mathcal{U}_{n}\left(n_{i j}\right)$ and that $W$ is an extension of $W \cap H$ for each $W \in \mathcal{U}_{n+1, H}\left(n_{i j}\right)$. Fix any $X \subset H$ such that $X \in \mathcal{U}_{n}\left(n_{i j}\right)$. Let $\rho: \mathcal{U}_{n+1, H}\left(n_{i j}\right) \rightarrow \mathcal{U}_{n}\left(n_{i j}\right)$ be the morphism defined by the formula $W \mapsto W \cap H$. There are exactly $\infty^{n+1} W \in \mathcal{U}_{n+1, H}\left(n_{i j}\right)$ such that $W \cap H=X$ and $W$ is a cone with vertex containing some $p \in \mathbb{P}^{n+1} \backslash H$. Thus $\rho$ is a surjective morphism between integral varieties. Thus if $W$ is general in $\mathcal{U}_{n+1}\left(n_{i j}\right)$, then $W \in \mathcal{U}_{n+1, H}\left(n_{i j}\right)$ and $W \cap H$ is general in $\mathcal{U}_{n}\left(n_{i j}\right)$. Recall that for each $X \in \mathcal{U}_{n}\left(n_{i j}\right)$ the set of cones belonging to $\rho^{-1}(X)$ has dimension $n+1$. Thus to prove that a general $X \in \mathcal{U}_{n}\left(n_{i j}\right)$ may be extended to a general $W \in \mathcal{U}_{n+1}\left(n_{i j}\right)$ which is not a cone with vertex contains some $p \in \mathbb{P}^{n+1} \backslash H$ it is sufficient to prove that $\psi_{n+1}\left(n_{i j}\right) \geq \psi_{n}\left(n_{i j}\right)+n+2$. If we do this for all large $n$ then we get an example for the case codimension 2 of Theorem 1.2. We only do one example of $s$ and $n_{i j}$, which satisfies these properties for all $n \geq 3$.

Fix integers $s \geq 3$ and an integer $c \geq 2$. Set $n_{1 i}:=(s-1) c$ for all $i$ and $n_{2 j}:=s c$ for all $j$. We have $\psi_{n}\left(n_{i j}\right)=s(s-1)\left(\begin{array}{c}x+n \\ n\end{array}\right)-(s-1)^{2}-s^{2}+1$. Thus $\psi_{n+1}\left(n_{i j}\right)-\psi_{n}\left(n_{i j}\right)=s(s-1)\left(\begin{array}{l}x+n \\ n+1\end{array}\right) \geq s(s-1)(n+2) / 2$. For $n=3$ this case corresponds to an integral curve because its numerical character is connected ([10, Th. 2.5]) and it is even smooth ([17]). Hence inductively we get an integral $X$, as claimed by the theorem.

(b) Now we do the codimension 3 case. In this part we get $X$ which is arithmetically Gorenstein. We use a paper by J. O. Kleppe and R.-M. Miró-Roig ([11), which of course use the classical Buchsbaum-Eisenbud's description of codimension 3 Gorenstein local rings ([2]). At the end of [11, §1] they introduce the integers appearing in their computation of the dimension of the Hilbert scheme of arithmetically Gorenstein codimension 3 subschemes of $\mathbb{P}^{n}, n \geq 4$, with prescribed numerical invariants $f$ (or $e$ with $f=e+n+1$ ), $r \geq 3, n_{2 i}$ and $n_{1 j}$ with $n_{11} \leq \cdots \leq n_{1 r}$, $n_{21} \geq \cdots \geq n_{2 r}$ with $n_{1 i}$ for all $i$. The dimension of this iirreducible component of the Hilbert scheme is given in [11, Remark 2.8]. Call $\varphi_{n}\left(n_{i j}\right)$ this dimension. By 
[1. Remark 2.8] we have

$$
\begin{aligned}
& \varphi_{n}\left(n_{i j}\right)=\sum_{1 \leq i<j \leq r}\left(\begin{array}{c}
-n_{1 i}+n_{2 j}+n \\
n
\end{array}\right) \\
& -\sum_{1 \leq i, j \leq r}\left(\begin{array}{c}
-n_{1 i}+n_{1 j}+n \\
n
\end{array}\right)+\sum_{1 \leq i \leq j \leq r}\left(\begin{array}{c}
n_{1 i}-n_{2 j}+n \\
n
\end{array}\right)
\end{aligned}
$$

We only give one case, the case with $r=7, n_{11}=n_{12}=n_{13}=n_{14}=n_{15}=4$, $n_{16}=n_{17}=n_{26}=n_{27}=5$ and $n_{21}=n_{22}=n_{23}=n_{24}=n_{25}=6$. The big advantage of this case is that we do not need to check the existence of an integral $X$, since in the starting case, the case $n=4$, we may take $X$ smooth and irreducible ([11, Example 2.9]. We have

$$
\begin{aligned}
& \varphi_{n}\left(n_{i j}\right)=10\left(\begin{array}{c}
-4+6+n \\
n
\end{array}\right)+10\left(\begin{array}{c}
-4+5+n \\
n
\end{array}\right)-25-4 \\
& -10\left(\begin{array}{c}
-4+5+n \\
n
\end{array}\right)+3=10\left(\begin{array}{c}
n+2 \\
2
\end{array}\right)-26
\end{aligned}
$$

Thus $\varphi_{n+1}\left(n_{i j}\right)-\varphi_{n}\left(n_{i j}\right)=10(n+2)>n+2$.

(c) Take any $x \geq 4$ and any $n \geq x+1$. By step (b) there are integral, nondegenerate, arithmetically Cohen-Macaulay and Gorenstein varieties $Y_{i} \subset \mathbb{P}^{n+i}$, $i \geq 0$, such that each $Y_{i}, i>0$, is an extension (not cone-like) of $Y_{i-1}$ and no $Y_{i}$ is a complete intersection. Fix general quadric hypersurfaces $Q_{1}(0), \ldots, Q_{x-3}(0) \subset \mathbb{P}^{n}$. Let $Q_{j}(r) \subset \mathbb{P}^{n+r}$ be an $r$-step extension of $Q_{j}(0), 1 \leq j \leq x-3$. Since $Q_{j}(0)$ is general, it is smooth. Thus we may find as extensions smooth quadric hypersurfaces. Set $X_{i}:=Y_{i} \cap Q_{1}(i) \cap \cdots \cap Q_{x-3}(i)$ and $X:=X_{0}$. Each $X_{i} \subset \mathbb{P}^{n+i}$ is nondegenerate, integral (by Bertini's theorem) and arithmetically Gorenstein. Since a minimal system of generators of the homogeneous ideal of $X_{i}$ is obtained adding $Q_{1}(i), \ldots, Q_{x-3}(i)$ to a minimal system of generators of $Y_{i}, X_{i}$ is not a complete extension. Fix an integer $i>0$ and let $H \subset \mathbb{P}^{n+i}$ be the hyperplane generated by $Y_{i-1} . H$ is the hyperplane generated by $X_{i-1}$. Assume the existence of $p \in \mathbb{P}^{n+i} \backslash H$ such that $X_{i}$ is a cone with vertex $p$ and $X_{i-1}$ as a basis. Since $X_{i-1}$ spans $H, \mathbb{P}^{n+i}$ is the Zariski tangent space to $X_{i}$ at $p$. Thus each quadric hypersurface containing $X_{i}$ is a cone with vertex $p$. Thus $Q_{1}(i)$ is a cone, a contradiction.

\section{REFERENCES}

1. W. Barth and A. Van de Ven, A decomposability criterion for algebraic 2-bundles on projective spaces, Invent. Math. 25 (1974), 91-106.

2. D. Buchsbaum and D. Eisenbud, Algebra structures for finite free resolutions, and some structure theorems for ideals of codimension 3, Amer. J. Math. 99 (1977) 447-485.

3. C. Ciliberto, T. Dedieu and E. Sernesi, Wahl maps and extensions of canonical curves and K3 surfaces, J. Reine Angew. Math. 761 (2020), 219-245.

4. I. Coandă, Infinitely stably extendable vector bundles on projective spaces, Arch. Math. 94 (2010), 539-545.

5. I. Coandă, A simple proof of Tyurin's Babylonian tower theorem, Comm. Algebra 40 (2012), no. $12,4668-4672$.

6. I. Coandă, and G. Trautmann, The splitting criterion of Kempf and the Babylonian tower theorem, Comm. Algebra 34 (2006), 2485-2488.

7. G. Ellingsrud, Sur le schéma de Hilbert des variétés de codimension 2 dans $\mathbf{P}^{e}$ à cône de Cohen-Macaulay, Ann. Sci. École Norm. Sup. (4) 8 (1975), no. 4, 423-431.

8. M. L. Fania, and E. L. Livorni, Degree ten manifolds of dimension n greater than or equal to 3, Math. Nachr. 188 (1997), 79-108. 
9. H. Flenner, Babylonian tower theorems on the punctured spectrum, Math. Ann. 271 (1985), $153-160$.

10. L. Gruson and C. Peskine,Genre des courbes de l'espace projectif, in: Algebraic geometry. Troms $\varnothing$ 1977. Leer. Notes Math. 687, 31-59. Berlin, Heidelberg, New York: Springer 1978

11. J. O. Kleppe and R.-M. Miró-Roig, The dimension of the Hilbert scheme of Gorenstein codimension 3 subschemes, J. Pure Appl. Algebra 127 (1998), 73-82.

12. S. Lvovsky, Extensions of projective varieties and deformations, I, II, Michigan Math. J. 39 (1992), 41-51, 65-70.

13. J. Migliore, Introduction to liaison theory and deficiency modules, Birkäuser, Boston-BaselBerlin, 1998.

14. I. B. Penkov and A.S.Tikhomirov, On the Barth-Van de Ven-Tyurin-Sato theorem, (Russian) Mat. Sb. 206 (2015), no. 6, 49-84; translation in Sb. Math. 206 (2015), no. 5-6, 814-848.

15. E. Sato, On the decomposability of infinitely extendable vector bundles on projective spaces and Grassmann varieties. J. Math. Kyoto Univ. 17 (1977), 127-150.

16. E. Sato, The decomposability of an infinitely extendable vector bundle on the projective space, II. In: International Symposium on Algebraic Geometry. Kyoto University. Kinokuniya Book Store: Tokyo, pp. 663-672, 1978.

17. T. Sauer, Smoothing projectively Cohen-Macaulay space curves, Math. Ann. 272 (1985), no. $1,83-90$.

18. H. P. F. Swinnerton-Dyer, An enumeration of all varieties of degree 4, Amer. J. Math. 95 (1973), 403-418.

19. A. L. Tironi, Normal projective varieties of degree 5, Comm. Algebra 42 (2014), no. 10, $4322-4332$.

20. A. N. Tyurin, A. N. Finite dimensional vector bundles over infinite varieties. Math. USSR Izv. 10 (1976), 1187-1204.

21. $\mathrm{XXX}=\mathrm{A}$. Weil, Correspondence by XXX, Amer. J. Math. 79 (1957), 951-952; reprinted in Oeuvres scientifiques/collected papers. II. 1951-1964, Appendix I, pp. 555-556.

22. F. L. Zak, Projections of algebraic varieties, Mat. Sb. (N.S.) 116(158) (1981), no. 4, 593-602, 608.

23. F. L. Zak, Some properties of dual varieties and their applications in projective geometry, Algebraic geometry (Chicago, IL, 1989), 273-280, Lect. Notes in Math. 1479, Springer, Berlin, 1991.

Dept. of Mathematics, University of Trento, 38123 Povo (TN), Italy

Email address: edoardo.ballico@unitn.it 